\title{
Notulae orthopterologicae \\ XXI - Le Dolichopoda della Francia e della Spagna
}

\author{
Par Bacio Bacgetti ${ }^{1}$ ) \\ Con tavole $5(1)-8(4)$
}

La recente revisione delle Dolichopoda italiane pubblicata nel 1959 da Baccetti e Capra, aveva aperto alcuni problemi sulla sistematica delle Dolichopoda francesi. In particolare la istituzione del lectotipo di Dolichopoda palpata Sulz., che veniva interpretata come specie presente soltanto nell'estrema Italia meridionale, richiedeva chiarimenti sulla posizione di quelle Dolichopoda francesi che Chopard (1951) denominava palpata, ma che verosimilmente avrebbero dovuto essere attribuite ad altra specie.

Da molto tempo, del resto, le Dolichopoda dell'Europa occidentale abbisognavano di una revisione nella quale venissero utilizzati i caratteri dell'epifallo e del decimo tergo dei maschi, rivelatisi basilari per la sistematica del genere. Tale revisione forma l'oggetto di questa nota, ed ha potuto compiersi grazie alla cortesia del prof. Lucien Chopard, che ha voluto affidarmi in studio l'intero materiale francese e spagnolo del Museum National d'Histoire Naturelle di Parigi, concedendomi anche in esame tipi di specie orientali che mi sono serviti per la elaborazione della chiave analitica dell'intero genere Dolichopoda. A lui vadano i miei più vivi ringraziamenti.

\section{DOLICHOPODA (s.str.) AZAMI Saulcy}

Dolichopoda Azami Saulcy in Azam, 1893, Ann. Soc. Ent. France, LXII, pp. 196-197 : Patria tipica : Grotte de Chauves Souris, Châteaudouble, Var.

Dolichopoda palpata var. melusinae Bernard, 1946, Ann. Un. Grenoble, 21, p. 434 .

Dolichopoda palpata ssp. azami Saulcy, Chopard, 1951, F. Fr., pp. 174-175. Dolichopoda azami Baccetti e Capra, 1959, Redia, XLIV, pp. 187-190.

Materiale esaminato: Châteaudouble (Var), 1888, 2 șơ e 1 q; Val-

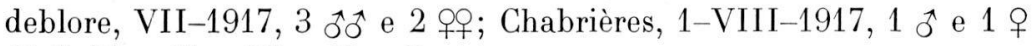
(Coll. Mus. Nat. Hist. Nat. Paris).

1) Stazione di Entomologia Agraria, via Romana 15/A, Firenze. 
L'esame di numerosi esemplari di entrambi i sessi e di molte località di questa specie, la cui area di diffusione appare, nella Francia sudorientale, assai ampia e raggiunge l'Italia occidentale presso Cuneo (Baccetti e Capra, 1959), mi ha convinto della scarsa importanza di un carattere tassonomico ritenuto tipico, e cioè della presenza di 25 denticoli apicali nell'ovopositore. Tale carattere è realmente mostrato solo dagli esemplari raccolti nella patria tipica della specie, nel Var, mentre manca nelle popolazioni di Valdeblore e di Chabrières, nelle quali il numero si mantiene sempre attorno a 18. Ovviamente la sicurezza della diagnosi è corroborata dalla contemporanea presenza di tutti gli altri caratteri di recente messi in evidenza (Baccetti e Capra, 1959) e si potrebbe al massimo invocare un incipiente differenziamento razziale. Quest'ultima ipotesi è confermata dalla presenza di una popolazione nell'Isère (Cuves de Sassenage, presso Grenoble) che per l'abbondante spinulazione delle tibie anteriori e medie è stata da Bernard (1946) descritta come una nuova varietà di D. palpata. Cadendo l'importanza dei 25 denticoli dell'ovopositore come carattere specifico di azami, si viene ad attribuire a questa specie la razza a 18 denticoli descritta dal Bernard (e di cui ho esaminato i tipi), incominciando così a tratteggiare per grandi linee il differenziamento subspecifico di Dolichopoda azami, specie ad ampia geonemia.

\section{DOLICHOPODA (s.str.) CHOPARDI n.sp.}

Dolichopoda palpata Chopard, F. Fr., 1951, p. 174.

Colorazione fondamentale del corpo testacea, con una fascia bruno scura ben evidente lungo il margine posteriore dei terghi toracici e addominali.

Spinulazione delle zampe priva di caratteristiche peculiari: i femori sono inermi, le tibie spinulose secondo la formula più diffusa nel genere:

$\begin{array}{lcccc} & \text { Marg. } & \text { Marg. } & \text { Marg. } & \begin{array}{c}\text { Marg. } \\ \text { sup. est. }\end{array} \\ \text { sup. int. } & \begin{array}{c}\text { inf. est. } \\ \text { inf. int. }\end{array} \\ \text { Anteriori } & 5-7 & 5-7 & 4-5 & 3-4 \\ \text { Medie } & 7 & 5-8 & 3-4 & 3 \\ \text { Posteriori } & 17-19 & 16-19 & 2-3 & 1\end{array}$

Maschio - Decimo tergo privo di tubercoli spiniformi, e con espansioni lobiformi del margine posteriore rotondeggianti, molto piccole e del tutto laterali. Ciascuna di esse infatti è larga circa $1 / 6$ dell'intervallo che separa le inserzioni dei cerci. Lo spazio che separa fra di loro le due espansioni suddette rappresenta più di metà della larghezza 
dell'intero tergo; quivi il margine appare privo di espansioni angolose ma sporgente leggermente all'indietro. Gli stili sono assai piccoli, lunghi circa $0,15 \mathrm{~mm}$ e larghi altrettanto.

Epifallo assai ben sclerificato e scuro, con processo dorsale mediano assai largo alla base ma bruscamente ristretto verso il terzo prossimale, indi snello, progressivamente ricurvo ed appuntito. Processi basali allungati, gli anteriori assai più brevi dei posteriori, tutti perpendicolari al processo mediano.

Femmina - Lamina sottogenitale a contorno subcircolare, con una prominenza emisferica centrale assai ampia, subapiçale, bordo in evidente rilievo e privo di interruzioni. Ovopositore robusto relativamente breve, con 17 denticolazioni.

\section{Dimensioni in $\mathrm{mm}$.}

$\begin{array}{lcc} & \text { Maschio } & \text { Femmina } \\ \text { Long. corp. } & 15 & 15 \\ \text { Long. pronoti } & 3,5 & 3,5 \\ \text { Long. fem. post. } & 20 & 20 \\ \text { Long. ovop. } & - & 11\end{array}$

Olotipo ô, Allotipo ㅇ, Villiers, 1700 m., Haute Tinée (Alpes Maritimes), VIII-1958 (Coll. Mus. Nat. Hist. Natur. Paris).

Osservazioni - Questa specie, che sembra propria delle montagne della alta Tinée, ad elevate quote, appartiene al gruppo azamiligustica, come documenta la assenza di tubercoli spiniformi sul $\mathrm{X}$ tergo del maschio. Per la forma dell'epifallo (pur essendo ben distinta da entrambe le specie) si avvicina maggiormente ad azami, ma per la forma generale del X tergo del maschio appare assai simile a ligustica. E'assai più piccola, e soprattutto più brachipoda, di entrambe le specie. Anche l'ovopositore appare più breve, e con numero di spinule (17) nettamente ridotto, e sensibilmente inferiore a quello dei più tipici esemplari di azami. Abbiamo però visto come in questa ultima in molti casi tale numero possa raggiungere quello di 18, più generalmente riscontrato nelle Dolichopoda.

Questa specie è stata segnalata in Francia, sempre nell'Alta Tinea, molto recentemente (Chopard, 1951), e giustamente avvicinata alle popolazioni italiane, ritenute tipica palpata. Ma essa appare ben differenziata, se pur chiaramente appartenente al medesimo gruppo di specie evolutesi sulle Alpi occidentali italiane e francesi. 


\section{DOLICHOPODA (s.str.) LINDERI Duf.}

Phalangopsis linderii Dufour, 1861, Ann. Soc. Ent. Fr. (4) I, p. 13 : Patria tipica : Grotte de Villefranche (Pirenei Orientali).

Dolichopoda linderi Brunner, 1882, Prodr. Eur. Orth., p. 413.

Dolichopoda linderi Chopard, 1951, F. Fr., p. 176.

Colorazione generale del corpo testacea, con una fascia bruno scura molto sottile lungo il margine posteriore di tutti i terghi. La spinulazione delle zampe presenta la sola caratteristica peculiare di mostrare talora una notevole riduzione nella mole e nel numero delle spinule apicali dei femori, che da 2-3 divengono una o nessuna, ma il carattere (da molti Autori preso come tipico) non è costante. A parte le spinule apicali, i femori sono totalmente inermi. Il numero delle spinule delle tibie varia entro i limiti seguenti:

$\begin{array}{lcccc} & \begin{array}{l}\text { Marg. } \\ \text { sup. est. }\end{array} & \begin{array}{c}\text { Marg. } \\ \text { sup. int. }\end{array} & \begin{array}{c}\text { Marg. } \\ \text { inf. est. }\end{array} & \begin{array}{c}\text { Marg. } \\ \text { inf. int. }\end{array} \\ \text { Anteriori } & 0-3 & 0-3 & 3-4 & 3-6 \\ \text { Medie } & 3-6 & 5-8 & 4-5 & 3-4 \\ \text { Posteriori } & 19-20 & 19-20 & 2 & 1-2\end{array}$

Maschio - Decimo tergo privo di tubercoli spiniformi. Espansioni lobiformi del margine posteriore poco sporgenti ma assai larghe, trasverse, misuranti ciascuna in larghezza quasi un terzo dello spazio compreso fra i cerci e separate da un tratto del margine posteriore dritto e piano. Lamina sottogenitale con incisione assai profonda, raggiungente quasi il margine prossimale della lamina stessa. Stili relativamente lunghi (quasi $1 \mathrm{~mm}$ ) e ben evidenti.

Epifallo di colore chiaro, non ben sclerificato, con processo dorsale mediano assai snello, progressivamente appuntito e scarsamente ricurvo. Processi basali posteriori perpendicolari al processo mediano; processi basali anteriori praticamente assenti.

Femmina - Lamina sottogenitale con contorno subcircolare, prominenza emisferica in netto rilievo, bordo ben rilevato e continuo, senza fossette apicali. Ovopositore lungo e robusto, munito di 21-25 denticolazioni.

\begin{tabular}{lcc}
\multicolumn{3}{c}{ Dimensioni in mm. } \\
& Maschio & Femmina \\
Long. corp. & 15 & 18 \\
Long. pronot. & 3 & 4 \\
Long. fem. post. & 21 & 26 \\
Long. ovop. & - & 14
\end{tabular}


In questa specie è evidente un dimegetismo sessuale particolarmente marcato.

Materiale esaminato: Grotte de Villefranche (Pirenei Orientali),


1 우 (Coll. Mus. Nat. Hist. Natur. Paris).

Osservazioni - Questa specie sembra limitata alle grotte della Francia Meridionale, dai Pirenei Orientali alle Cevenne. Ben nota fino dallo scorso secolo, si dimostra nettamente distaccata dalle Dolichopoda alpine per la forma dell'epifallo, privo di processi basali anteriori ben sviluppati, e del $\mathrm{X}$ tergo del maschio: caratteri che invece, come vedremo, la ravvicinano molto alla specie iberica D. bolivari Chop.

\section{DOLICHOPODA (s.str.) BOLIVARI Chop.}

Dolichopoda bolisari Chopard, 1915, Bull. Soc. Ent. Fr., p. 276 : Patria tipica : Forat negre, Lerida, Spagna.

Dolichopoda bolivari Chopard, 1922, F. Fr., p. 92.

Dolichopoda bolivari Chopard, 1951, F. Fr., p. 175.

Colarazione testaceo chiara con margine posteriore dei terghi sottilmente fasciato di bruno. La spinulazione delle zampe risponde alla formula più consueta nelle Dolichopoda s.str. I femori sono cioè inermi, e soltanto muniti di $2-3$ spinette apicali; le tibie armate di spinule il cui numero varia entro i limiti seguenti:

$\begin{array}{lcccc} & \text { Marg. } & \text { Marg. } & \text { Marg. } & \text { Marg. } \\ & \text { sup. est. } & \text { sup. int. } & \text { inf. est. } & \text { inf. int. } \\ \text { Anteriori } & 2-4 & 2-4 & 3-4 & 3-4 \\ \text { Medie } & 5-7 & 4-6 & 2-3 & 2-3 \\ \text { Posteriori } & 17-18 & 16-19 & 0-2 & 0-1\end{array}$

Maschio - Decimo tergo privo di tubercoli spiniformi. Espansioni lobiformi del margine posteriore pochissimo sporgenti, larghe un terzo dello spazio compreso fra i cerci e separate da un tratto del margine completamente piano e non rilevato. Incisione della lamina sottogenitale raggiungente il margine prossimale della lamina. Stili lunghi quasi $1 \mathrm{~mm}$.

Epifallo ben sclerificato, con processo dorsale mediano snello, progressivamente ricurvo ed appuntito. Processi basali posteriori perpendicolari al processo mediano; processi basali anteriori troncati presso la base, talora prolungati in due brevi punte rivolte dorsalmente.

Femmina - Lamina sottogenitale trasversa, assai più larga che lunga, a contorno semicircolare, con prominenza emisferica e bordo interrotto da una fossetta apicale. 
Materiale esaminato: Cueva Forat Negre, Serradell (Lerida) 18VIII-1918, 1 ô e 3 of (Bolivar leg.); Forat de la Bou, Serradell (Lerida), 16-VIII-1911, 1 o̊ e 2 우 (Bolivar leg.); Cueva Carbonera, C. de Pont de Suert, 12-VIII-1918, 1 ô et 1 o; Forat de les Tables (Lerida), 23-VIII-1918, 4 neanidi e 1 o ad. (S. Maluquer leg.); Cava del Forti, Serradell (Lerida), 3 ỡ e 1 q; Cova Roya de Sta, C. de Pont de Suert, molte neanidi (Coll. Mus. Nat. Hist. Natur. Paris).

Osservazioni - Questa specie, assai affine alla D. linderi della Francia meridionale, sembra propria della penisola Iberica, ove è la sola Dolichopoda nota. Per ora appare limitata alle grotte della Catalogna.

\section{Chiave analitica del genere Dolichopoda}

1. Femori posteriori inermi lungo il margine inferiore (subg. Dolichopoda).

2. Stili presenti.

3. Epifallo troncato all'apice, placca sottogenitale della femmina smarginata all'apice ..................... D. euxina Semenov 1901

(Caucaso)

3.3. Epifallo acuto, placca sottogenitale delle femmina arrotondata all' apice.

4. Terghi di tutto il corpo testacei, fasciati di bruno lungo il margine posteriore.

5. IXo tergo del maschio armato di un processo mediano lungo e stretto D. insignis Chopard $\mathbf{1 9 5 5}$

(Grecia: Attica)

5.5. IX o tergo del maschio privo di processi.

6. Tubercoli del $\mathrm{X}^{0}$ tergo del maschio assenti o rudimentali.

7. Epifallo bifido all'apice ........... D. petrochilosi Chopard 1954

(Grecia: Attica e Peloponneso Merid.)

7.7. Epifallo semplicemente acuto all'apice.

8. $\mathrm{X}^{\circ}$ tergo del maschio con due sole espansioni lobiformi del margine posteriore, del tutto laterali.

9. Espansioni lobiformi del $X^{0}$ tergo del maschio assai sporgenti, separate da una profonda concavità. Ovopositore con meno di 18 denticolazioni apicali.

10. Margini superiori delle tibie anteriori inermi. Ovopositore con 16-17 denticoli ..................... D. baccettii Capra 1957

(Italia, Toscana: M. Argentario)

10.10. Margini superiori delle tibie anteriori armati di 3-4 spinule. Ovopositore con 15 denticoli ................ D. graeca Chopard 1964

(Grecia: Epiro)

9.9. Espansioni lobiformi del $X^{0}$ tergo del maschio poco sporgenti, separate da una concavità assai modesta. Ovopositore con più di 18 denticolazioni apicali.

11. Espansioni lobiformi del $\mathrm{X}^{0}$ tergo del maschio assai larghe, ciascuna circa $1 / 3$ dello spazio compreso fra i cerci. Lamina sottogenitale del 
maschio con incisione assai profonda, raggiungente quasi il margine prossimale della lamina stessa.

12. Epifallo privo di processi basali anteriori. Ovopositore con 21-25 denticolazioni apicali ................... D. linderi Dufour 1861

(Francia: Pirenei orientali e Cevenne)

12.12. Epifallo con processi basali anteriori brevissimi e rivolti dorsalmente. Ovopositore con 18 denticolazioni apicali D. bolisari Chopard 1915

(Spagna: Catalogna)

11.11. Espansioni lobiformi del $\mathrm{X}^{0}$ tergo del maschio assai strette (ciascuna circa $1 / 6$ dello spazio compreso fra i cerci) e del tutto laterali. Lamina sottogenitale del maschio con incisione raggiungente circa la metà lunghezza.

13. Stili rudimentali, isodiametrici. Epifallo snello ed allungato.

14. Processo dorsale mediano dell'epifallo, visto di fronte, con una brusca costrizione a metà lunghezza. Intervallo fra i lobi del $X^{\circ}$ tergo presentante due piccole espansioni angolose sporgenti posteriormente ...... ................................. D. azami Saulcy 1893

(Francia sud-orientale; Piemonte: Cuneo)

14.14. Processo dorsale mediano dell'epifallo, visto di fronte, con una brusca costrizione presso il terzo prossimale. Intervallo fra i lobi del $\mathrm{X}^{\mathrm{o}}$ tergo privo di espansioni angolose .... D. chopardi Baccetti 1965

(Francia: Alpi Marittime)

13.13. Stili due volte più lunghi che larghi. Epifallo tozzo e corto ........ D. ligustica Baccetti e Capra 1959 (Italia: Piemonte e Liguria)

15. Epifallo con lobi della porzione anteriore della base corti e tozzi. Forma generale del pezzo molto tozza, colore bruno scuro ............

D. ligustica ssp. ligustica Baccetti e Capra 1959

(Italia: Liguria)

15.15. Epifallo con lobi della porzione anteriore della base stretti e lunghi. Forma generale del pezzo più snella, colore chiaro ............ ......... D. ligustica ssp. septentrionalis Baccetti e Capra 1959

(Italia, Piemonte: Val di Lanzo)

8.8. Decimo tergo del maschio con tre vistose espansioni lobiformi del margine posteriore, delle quali le due laterali sono triangolari e la centrale, meno sporgente, è quadrata . . D. thasosensis Chopard 1964

6.6. Tubercoli del $\mathrm{X}^{\text {o }}$ tergo del maschio ben evidenti.

(Grecia: Isola Thasos)

16. Tubercoli del $X^{0}$ tergo del maschio molto grandi, arrotondati, salienti ........................ D. araneiformis Burmeister 1838

(Dalmazia, Erzegovina)

16.16. Tubercoli del $\mathrm{X}^{0}$ tergo del maschio piccoli, appuntiti, spiniformi.

17. $\mathrm{X}^{\text {o }}$ tergo del maschio con margine posteriore fortemente concavo nella zona fra i due lob ilaterali, i quali sporgono vistosamente dal margine e costituiscono la quasi totalità dello sclerite D. hussoni Chopard 1934

(Macedonia)

17.17. Xo tergo del maschio con margine posteriore quasi dritto nella zona fra i due lobi laterali, i quali sporgono modestamente da un'ampia base che costituisce gran parte dello sclerite. 
18. Epifallo con una caratteristica costrizione alla base del processo mediano, che risulta a forma di freccia ......... D. geniculata Costa 1860 (Italia: Toscana meridionale, Abruzzi,Campania, Lucania e Calabria sett. ${ }^{2}$ )

18.18. Epifallo privo di costrizioni, regolarmente conico.

19. Espansioni lobiformi del $X^{0}$ tergo del maschio non divergenti ....... D. laetitiae Menozzi 1920

(Italia: Emilia, Toscana appenninica, Marche, Umbria, Lazio)

19.19. Espansioni lobiformi del $\mathrm{X}^{0}$ tergo del maschio divergenti ........ D. palpata Sulzer 1776

(Italia: Sicilia, Calabria merid.)

4.4. Colorazione di tutto il corpo uniformemente testacea senza fascia bruna lungo il margine posteriore dei terghi D. unicolor Chopard 1964

2.2. Stili assenti.

(Albania)

20. Statura maggiore (pronoto $5,5 \mathrm{~mm}$., fem. post. $24,5 \mathrm{~mm}$., ovopositore $13 \mathrm{~mm}$. D. patrizii Chopard 1964 (Grecia: Isola Petalas)

20.20. Statura minore (pronoto $3 \mathrm{~mm}$., fem. post. $23 \mathrm{~mm}$., ovopositore $10 \mathrm{~mm}.) \quad \ldots \ldots \ldots \ldots \ldots \ldots \ldots \ldots \ldots \ldots \ldots \ldots \ldots$ D. aranea Bolivar $1899^{3}$ )

(Asia Minore)

1.1. Femori posteriori armati di numerosissime spine lungo i margini inferiori ....................... (subg. Chopardina)

21. Femori anteriori inermi lungo i margini inferiori.

22. $X^{0}$ tergo del maschio munito di due piccoli tubercoli ..............

D. schiapazzii Capra 1934

(Italia: Toscana litoranea)

23. Femori medi abbondantemente spinulosi lungo i margini inferiori ....

D. schiapazzii ssp. schiapazzii Capra 1934

(Italia: Toscana litoranea)

23.23. Femori medi inermi o muniti di rare spinule lungo il margine inferiore ................... D. schiavazzii ssp. caprai Lanza 1954

(Italia, Toscana: Bagni di Casciano)

\footnotetext{
$\left.{ }^{2}\right)$ Baccetti e Capra (1959) consideravano come Dolichopoda geniculata solo gli semplari dell'Abruzzo meridionale, Campania, Lucania, e Calabria sett., ed istituivano una nuova specie (D. etrusca) per una popolazione leggermente diversa ed isolata della Toscana meridionale (Grosseto). L'esame di ulteriore materiale di $D$. geniculata proveniente tanto da tutto l'Abruzzo (Grotta delle Panie, Lettomanoppello, XII-1962 ; Grotta presso Opi, l'Aquila; Grotta La Fossa, Rapino, Chieti) che dalla Campania (sotterranei di Nocera inferiore, 18-XII-1946), nei quali l'epifallo mostra condizioni assai simili a quelle ritenute tipiche di etrusca, mi convince a considerare Dolichopoda etrusca Baccetti e Capra 1959 come sinonimo di D. geniculata Costa 1860. La popolazione toscana potrà al massimo essere considerata una razza geografica di geniculata, allorchè l'esame di più abbondante materiale consentiza una sicura valutazione del campo di variabilità della costrizione basale dell'epifallo.

$\left.{ }^{3}\right)$ I caratteri sono ricavati dalla breve descrizione di Bolivar (1899), poichè la specie, che sembra endemica dell-Asia minore, non è stata mai più raccolta in seguito.
} 
22.22. Decimo tergo del maschio privo di tubercoli D. remyi Chopard 1934

(Macedonia)

21.21. Femori anteriori armati di numerose spine lungo i margini inferiori.

24. Stili un poco allungati, placca sottogenitale della femmina triangolare D. cyrnensis Chopard 1950

(Corsica)

24.24. Stili sferici, placca sottogenitale della femmina semicircolare ...... D. bormansi Brunner 1882

(Corsica)

\section{RIASSUNTO}

La revisione delle Dolichopoda franco-iberiche ha condotto alla identificazione nella zona di quattro diverse specie di Dolichopoda s.str.; di queste una (D.azami) sembra avere una ampia geonemia sulle Alpi francesi, ed era già stata segnalata anche in Italia; una seconda, nuova per la scienza (D. chopardi m.) appare limitata alle Alpi marittime; la terza e la quarta (D. linderi e $D$. bolivari) già ben note, sembrano limitate ai Pirenei e alle Cevenne.

Dolichopoda azami e chopardi per i loro caratteri morfologici vengono a costituire un gruppo molto omogeneo, comprendente anche la D. ligustica, che potremmo definire alpino; $D$. linderi e bolivari sono invece ben diverse da queste ed affini fra di loro. Tale affinità, del resto, emergeva anche in parte dalle osservazioni citologiche di Saltet $(1959,1960)$. Al gruppo alpino potremmo cioè contrapporre un gruppo pirenaico. Si conferma cosi che l'isolamento geografico ha avuto una importanza basilare per il differenziamento specifico delle Dolichopoda franco-iberiche, che sembrano tutte derivate da un unico, antichissimo ceppo comune.

La elaborazione della chiave analitica che chiude il lavoro documenta tale affinità, e svela invece una situazione assai più complessa in Italia ed in Grecia, ove possono essersi verificate due diverse immigrazioni successive. La presenza in entrambe le penisole dei due diversi sottogeneri Dolichopoda e Chopardina (il secondo più antico del primo: Baccetti, 1960) lo conferma in pieno.

Complessivamente sono note quattro Chopardina (una in Macedonia, una in Toscana, due in Corsica) e 19 Dolichopoda, delle quali una caucasica, una anatolica, quattro distribuite fra Attica, Peloponneso e isole minori della Grecia, una macedone, tre nella costa balcanica fra la Dalmazia e l'Epiro, tre appenniniche, una praticamente localizzata nell'arcipelago toscano, tre alpine e due pirenaiche.

Si può dire che gli elementi di ciascun gruppo sono legati da strette affinità, cui costituiscono solo apparenti eccezioni fenomeni di parallelismo morfologico a grande distanza: ad esempio fra Dolichopoda baccettii e Dolichopoda graeca. Poichè, allorchè non vi siano condizioni di insularità che per il particolarmente marcato differenziamento specifico di molte entità sembrano essere state determinanti, le barriere geografiche fra gli elementi di ciascun gruppo sono spesso assai esigue, si può ritenere che dopo le due grandi immigrazioni da oriente che importarono uno dopo l'altro i due sottogeneri nel corso del Terziario (Baccetti, 1960) i fenomeni di speciazione siano stati largamente favoriti, nel corso del Quaternario, dalla acquisizione della troglofilia, che ha molto ostacolato le possibilità di migrazione. 


\section{SUMMARY}

The revision of French-Iberian Dolichopoda has led to the identification of four different species of Dolichopoda s. str. One of these (D.azami) seems to have a large geonomy in the French Alps and it had already been noticed in Italy too. A second species (D.chopardi m.), still new to science, seems to be restricted to the Maritime Alps; the third and the fourth (D.linderi and D.bolivari), already well known, seem to be localized in the Pyrenees and the Cevennes.

Owing to their morphological characters Dolichopoda azami and chopardi constitute a very homogeneous group, comprising also D.ligustica, and which could be defined alpine; on the contrary, D.linderi and bolivari are very different from said group and are very similar between them. Such similarity partly emerged from the citological observations of Saltet (19591960). We could then set an Alpine group against a Pyrenean one. It is then possible to state that the geographic isolation has a basic importance for the specific differentiation of the French-Pyrenean Dolichopoda, which appear to have descended from a unique, very old, common stock.

The elaboration of the analytical key closing the work, shows proof of such affinity and reveals a more complex situation in Italy and Greece where two successive, different immigrations may have taken place. Such theory is fully confirmed by the presence of two subgenera in both peninsulas, that is Dolichopoda and Chopardina (the second having preceded the first: Baccetti 1960). On the whole four Chopardina are known (one in Macedonia, one in Tuscany, two in Corsica) and nineteen Dolichopoda, one of which is caucasian, one anatolian, four distributed among Attica, Peloponnesus and minor islands of Greece, one in Macedonia, three are localized in the coast between Dalmatia and Epirus, three in the Appennin, one practically restricted to the Tuscan Archipelago, three in the Alps and two in the Pyrenees.

We could state that the elements of each group have close affinity and that the phenomena of morphological parallelism at great distance are but apparent exceptions: for instance Dolichopoda baccettii and Dolichopoda graeca. As - when there are no conditions of insularity causing a particularly remarkable differentiation specific to many entities - the geographic barriers among the elements of each group are often very scanty, we can consider possible that after the two great immigrations from the East, by which two subgenera, one after the other, were imported during the Terziary period (Baccetti, 1960), the phenomena of speciation were largely favoured, in the Quaternary, by the acquisition of troglophilia which has greatly hindered any possible migration.

\section{B I B LI OGRAF I A}

Azam, J. (1893) - Liste der Orthoptères des Hautes et Basses Alpes. - Ann. Soc. Ent. France, LXII, pp. 185-198.

Baccetti, B. (1958) - Notulae orthopterologicae. IX. Osservazioni cariologiche sulle Dolichopoda italiane. - Redia, XLIII, pp. 315-327, 6 figg.

- (1960) - I caratteri dell'ortotterofauna cavernicola italiana. - Atti Acc. Naz. It. Ent., Rend., VIII, 1961, pp. 317-323, 4 figg.

Baccetti, B., e Capra, F. (1959) - Notulae orthopterologicae. XII. Revisione delle specie italiane del genere Dolichopoda Bol. (Orthopt. Rhaphidophoridae). - Redia, XLIV, pp. 165-217, 21 figg. 
Bernard, F. (1948) - Observations faunistiques et biologiques aux Caves de Sassenage. - Travaux Lab. Hydrobiol. Piscic. Grenoble, 37-40, pp. 23-37, 3 tavv.

Bolivar, I. (1899) - Orthoptères du voyage de M. Martinez Escalera dans l'Asie mineure. - Ann. Soc. Ent. Belgique, XLIII, pp. 583-607.

Brunner von Wattenwyl, C. (1882) - Prodromus des europäischen Orthopteren. - Leipzig, Engelmann, XX +488 pp., 11 tavv., 1 carta.

Capra, F. (1934) - Una nuova Dolichopoda d'Italia. - Boll. Soc. Entom. Ital., LXVI, 3, pp. 40-43, 3 figg.

- (1957) - Una nuova Dolichopoda del monte Argentario. - Boll. Soc. Entom. Ital., LXXXVII, 5-6, pp. 90-92, 3 figg.

Chopard, L. (1915) - Diagnoses d'Orthoptères cavernicoles nouveaux (Stenopelmatidae). - Bull. Soc. Ent. France, pp. 276-279.

- (1917) - Notes sur deux espèces du genre Dolichopoda Bol. - Bull. Soc. Ent. France, n. 16, pp. 287-289.

- (1922) - Faune de France. 3. Orthoptères et Dermaptères. - Paris, Lechevalier, 212 pp., 466 fig., 1 tav.

- (1934) - Diagnoses d'Orthoptères cavernicoles nouveaux. - Bull. Soc. Ent. France, XXXIX, n. 9, pp. 137-139.

- (1936) - Biospeleologica N. LXIII. Orthoptères et Dermaptères (Première serie). - Arch. Zool. expér. gen., Paris, 78, f. 5, pp. 195-214.

- (1940) - Orthoptères cavernicoles recueillis par M. P. Remy en Yougoslavie et Macédonie. Comparaison entre la distribution des Orthoptères et des Coléoptères cavernicoles d'Europe. - VI. Congreso internacional de Entomologia, Madrid 1935, pp. 509-514, 2 carte.

- (1950) - Notes sur les Dolichopodes de Corse. - Bull. Soc. Ent. France, LV, n. 1, pp. 12-15, 5 figg.

- (1951) - Faune de France. 56. Orthoptéroides. - Paris, Lechevalier, $359 \mathrm{pp}, .531$ figg.

- (1954) - Contribution à l'étude des Orthoptéroides cavernicoles. - Notes Biospéléologiques, IX, pp. 27-36, 7 figg.

- (1955) - Les Dolichopodes de Grèce. - Notes Biospéléologiques, X, 1955, pp. 31-34, 3 figg.

- (1957) - Note sur les Orthoptères cavernicoles de Crète. - Bull. Soc. Ent. France, 62, pp. 26-28, 3 figg.

Karaman, Z. (1958) - Die Höhlenorthopteren Jugoslawiens. - Jahrb. Landu. Forstwirtschaft. Fak. Univ. Skopje, XI, pp. 211-238, 34 figg.

Lanza, B. (1957) - Speleofauna toscana. IV. Nuove notizie sulla Dolichopoda schiavazzii Capra. - Monit. Zool. Ital., LXIV (1956), n. 4, pp. 159-167, 1 fig.

Minozzi, C. (1920) - Nota complementare alla topografia e alla fauna della grotta di S. Maria M. sul monte Vallestra. - Atti Soc. Nat. Mat. Modena, ser. V, vol. V, pp. 71-74.

Saltet, P. (1959) - La formule chromosomique de Dolichopoda linderi Duf. (Orth. Rhaphidophoridae). - Compt. Rend. Sé. Acad. Scien., t. 248, pp. 851-853.

- $(1960)$ - La formule chromosomique de Dolichopoda palpata et D. bolivari (Orth. Rhaphidophoridae). - Compt. Rend. Sé. Acad. Scien., t. 250, pp. 2612-2614.

Semenov, A. (1901) - Un représentant cavernicole du genre Dolichopoda Bol. - Rev. Russe d'Entom., I, pp. 1-4. 


\section{SPIEGAZIONI DELLE TAVOLE $5(1)-8(4)$}

Fig. I

Dolichopoda azami Saulcy (Châteaudouble, Var). - Decimo tergo del maschio (1) ed epifallo, visto di fronte (2).

Fig. II

Dolichopoda chopardi m. (Villiers, Haute Tinée). - Decimo tergo del maschio (1), epifallo di lato (2) e di fronte (3).

Fig. III

Dolichopoda linderi Duf. (Grotte de Villefranche, Pirenei). - Decimo tergo del maschio (1), epifallo di lato (2) e di fronte (3).

Fig. IV

Dolichopoda bolivari Chop. (Forat Negre, Lerida). - Decimo tergo del maschio (1), epifallo di lato (2) e di fronte in due diversi esemplari(3 e 4).

Fig. V

Decimo tergo del maschio in Dolichopoda azami Saulcy (1) e D.ligustica Bacc. e Capra (2) (da Baccetti e Capra, 1959).

Fig. VI

Dolichopoda ligustica ligustica Bacc. e Capra. - Epifallo di fronte e di lato (da Baccetti e Capra, 1959).

Fig. VII

Dolichopoda geniculata Costa. - Epifallo di fronte (da Baccetti e Capra, 1959).

Fig. VIII

Decimo tergo del maschio in Dolichopoda hussoni Chop. (1), D.geniculata Costa (2), D. laetitiae Minozzi (3), D. palpata Sulz. (4) (il primo disegno è originale, gli altri tre sono ripresi da Baccetti e Capra, 1959). 
SPELEOLOGY II

(Baccetti, 1)

PLATE 5


II 

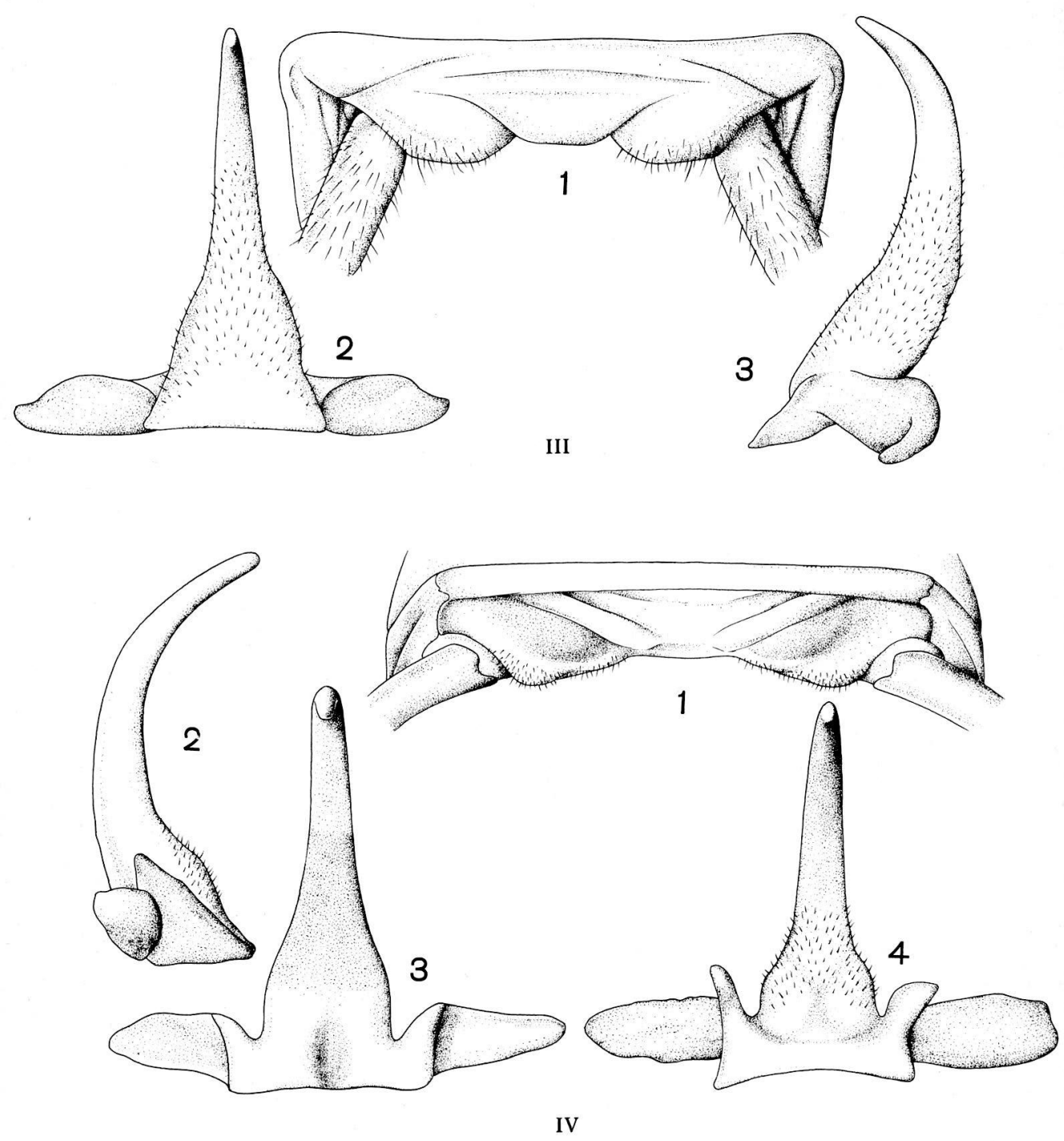


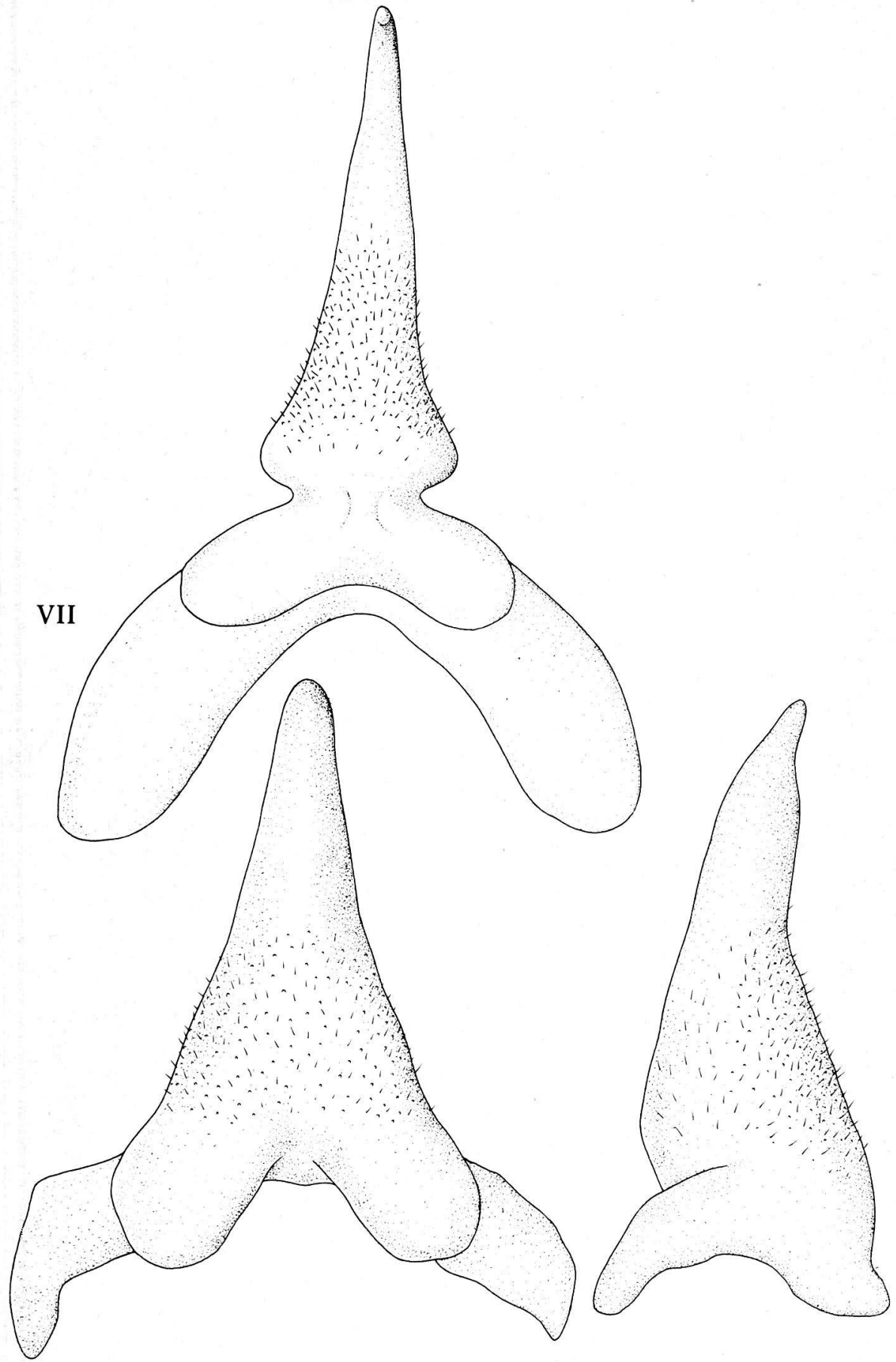




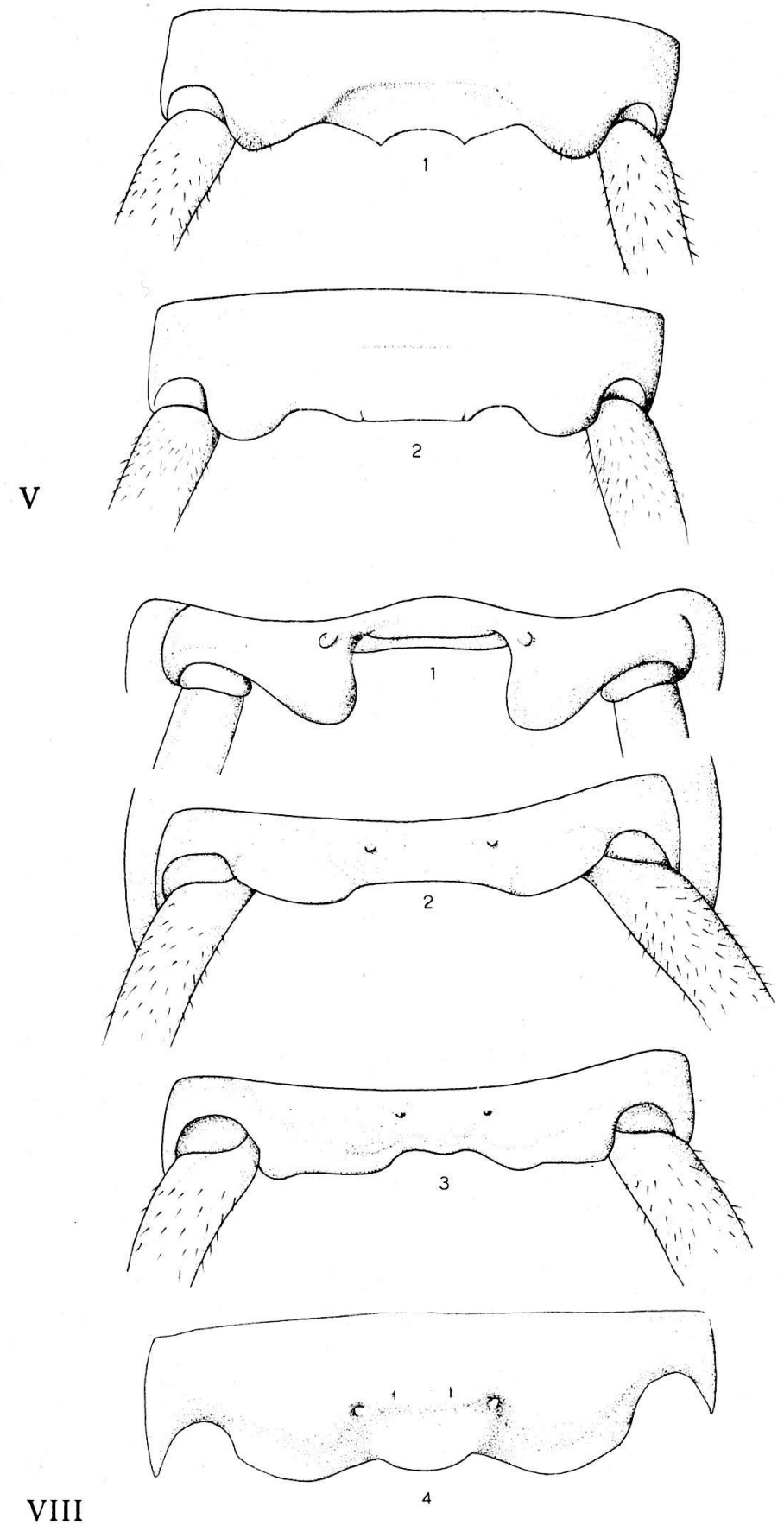

